\title{
Mass sensor utilising the mode-localisation effect in an electrostatically-coupled MEMS resonator pair fabricated using an SOI process
}

\author{
Graham S. Wood ${ }^{\mathrm{a}, *}$, Chun Zhao ${ }^{\mathrm{a}}$, Suan Hui Pu ${ }^{\mathrm{a}, \mathrm{b}}$, Stuart A. Boden ${ }^{\mathrm{a}}$, Ibrahim \\ Sari $^{\mathrm{a}}$, Michael Kraft ${ }^{\mathrm{c}}$ \\ ${ }^{a}$ Nano Research Group, School of Electronics and Computer Science, University of \\ Southampton, Mountbatten Building, Highfield Campus, Southampton, SO17 1BJ, U.K. \\ ${ }^{b}$ University of Southampton Malaysia Campus, No. 3 Persiaran Canselor 1, Kota Ilmu, \\ EduCity@Iskandar,79200 Nusajaya, Johor, Malaysia \\ ${ }^{c}$ Montefiore Institute, University of Liège, Building B28, Allée de la découverte 10, 4000 \\ Liège, Belgium
}

\begin{abstract}
The change in the mass, achieved with focused ion beam (FIB) milling, of one of a pair of electrostatically-coupled microelectromechanical systems (MEMS) resonators has been detected utilising the mode-localisation effect. It has been demonstrated that the shift in the amplitude ratio of the coupled-resonators at the in-phase mode-frequency, in response to a mass change, is five orders of magnitude greater than the equivalent resonant frequency shift of a single resonator device. The device has been fabricated using a silicon-on-insulator (SOI) based process, which allows for high-yield and stiction-free fabrication. In addition, the design of the resonators has been created to have a larger surface area than previously reported designs, in order to facilitate future biological functionalisation. The mass sensitivity has been compared to current state-ofthe-art mode-localised mass sensors and a 5.4 times increase in the amplitude ratio response to a given mass change has been demonstrated for the device in this work.
\end{abstract}

Keywords: MEMS, Resonators, Mass sensing, Mode-localization

\footnotetext{
${ }^{*}$ Corresponding author

Email address: gsw1g11@ecs.soton.ac.uk (Graham S. Wood)
} 


\section{Introduction}

The use of microelectromechanical systems (MEMS) structures for mass sensing is well established, with resonant frequency-shift being the predominant method of detection. Previous research has shown that a change in the mass 5 of a MEMS resonator can be quantified by detecting a shift in the resonant frequency [1]. The adsorption of gold atoms onto a nanoscale silicon carbide ( $\mathrm{SiC})$ clamped-clamped beam has been detected successfully through resonant frequency-shift [2]. Other studies have demonstrated the resonant detection of single-stranded DNA (ssDNA) probes [3] and Vaccinia virus particles [4] immobilised on microstructures. The detection of various biological elements is a widely researched area of resonant mass sensing with functionalised surfaces promoting the attachment of the target analytes. For example, the growth of Escherichia Coli [5] and the adsorption of spores of Bacillus anthracis [6] and Listeria innocua [7] on the surface of a cantilever have been demonstrated previously.

An alternative method of mass sensing exploits the mode-localisation effect in an array of two or more coupled resonators. If two closely spaced MEMS resonators are DC biased with different voltages, an electric field is created between them that acts as a coupling spring. If the mass of one of the resonators 20 is altered, the vibration amplitude ratio of the coupled system at the resonant mode frequencies shifts. The amplitude ratio shift occurs as a result of the confinement, or "localisation", of vibration energy in the now unbalanced coupled system [8. It has been reported previously that the response of the normalised mode shapes to a mass increase on one resonator in a coupled pair is three orders of magnitude greater than the response of the resonant frequency of a single resonator [9] and can be increased further by using a weaker coupling spring.

In this work, a coupled resonator device has been fabricated using a previously reported fabrication process [10]. This work details the mode-localisation behaviour of a pair of electrostatically-coupled resonators of a design architec- 


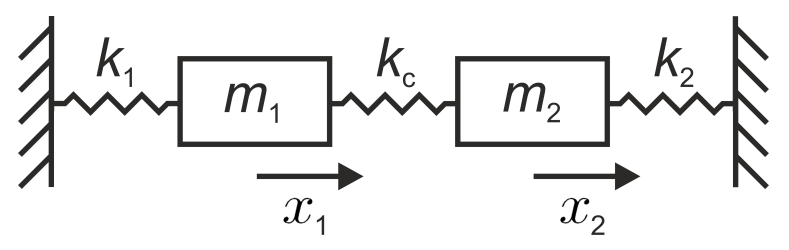

Figure 1: Mass-spring model of a coupled resonator system.

ture that differs from other work, which has characterised double-ended tuning forks and wine glass ring resonators [9]. Using a focused ion beam (FIB), the mass of one of the resonator pair has been reduced and the response of the in-phase resonant mode shape has been measured and the mass sensitivity of the device has been determined.

\section{Theory}

An analysis of a two degree-of-freedom (2-DOF) coupled resonator system has been performed with a simple lumped-element model, as shown in Fig. 1 . with a resonator modelled as a mass, $m_{1}$ or $m_{2}$, and a stiffness, $k_{1}$ or $k_{2}$. A

40 spring, $k_{c}$, provides the coupling element with the displacements of the resonators given by $x_{1}$ and $x_{2}$.

Beginning with the following equations of motion, the vibration response of the system can be derived

$$
\begin{aligned}
& m \ddot{x}_{1}+k x_{1}+k_{c}\left(x_{1}-x_{2}\right)=0 \\
& m \ddot{x}_{2}+k x_{2}+k_{c}\left(x_{2}-x_{1}\right)=0
\end{aligned}
$$

where $m_{1}=m_{2}=m$ and $k_{1}=k_{2}=k$ as the two resonators are mechanically 45 identical.

The equations of motion are converted into matrix form and expressed as an eigenvalue problem as follows

$$
A u_{i}=\lambda_{i} I u_{i}
$$


where $\lambda_{i}$ and $u_{i}(\mathrm{i}=1,2)$ represent the eigenvalues and eigenvectors respectively. $\mathrm{A}$ is given by

$$
\begin{aligned}
A & =M^{-1} K \\
& =\left[\begin{array}{cc}
\frac{1}{m} & 0 \\
0 & \frac{1}{m}
\end{array}\right]\left[\begin{array}{cc}
k+k_{c} & -k_{c} \\
-k_{c} & k+k_{c}
\end{array}\right] \\
& {\left[\begin{array}{cc}
\frac{k+k_{c}}{m} & -\frac{k_{c}}{m} \\
-\frac{k_{c}}{m} & \frac{k+k_{c}}{m}
\end{array}\right] }
\end{aligned}
$$

The eigenvalue problem is solved and the resulting eigenvalues $\left(\lambda_{i}=\omega_{i}{ }^{2}\right)$ and eigenvectors represent the mode frequencies and mode shapes, respectively, for the in-phase and out-of-phase modes, as follows

$$
\begin{gathered}
\lambda_{1}=\frac{k}{m}, u_{1}=\frac{1}{\sqrt{2}}\left[\begin{array}{l}
1 \\
1
\end{array}\right] \\
\lambda_{2}=\frac{k+2 k_{c}}{m}, u_{2}=\frac{1}{\sqrt{2}}\left[\begin{array}{c}
1 \\
-1
\end{array}\right]
\end{gathered}
$$

Next, using a previously described method [11], the response of the amplitude ratio at the two mode frequencies to a change in the mass, $m_{1}$, of resonator 1 has been derived. The following expressions give the amplitude ratio at the out-of-phase and the in-phase modes, where $o p$ and $i p$ denote the out-of-phase and in-phase modes, respectively

$$
\begin{aligned}
& \frac{X_{1}\left(j \omega_{o p}\right)}{X_{2}\left(j \omega_{o p}\right)}=\frac{1}{k_{c}}\left[k_{c}-k+\frac{m_{2}}{2}\left(\frac{\left(k-k_{c}\right)\left(m_{1}+m_{2}\right)}{m_{1} m_{2}}\right.\right. \\
& \left.\left.-\sqrt{\frac{\left(k-k_{c}\right)^{2}\left[\left(m_{1}+m_{2}\right)^{2}+4 m_{1} m_{2}\right]-4 m_{1} m_{2} k_{c}^{2}}{\left(m_{1} m_{2}\right)^{2}}}\right)\right] \\
& \frac{X_{1}\left(j \omega_{i p}\right)}{X_{2}\left(j \omega_{i p}\right)}=\frac{1}{k_{c}}\left[k_{c}-k+\frac{m_{2}}{2}\left(\frac{\left(k-k_{c}\right)\left(m_{1}+m_{2}\right)}{m_{1} m_{2}}\right.\right. \\
& \left.\left.+\sqrt{\frac{\left(k-k_{c}\right)^{2}\left[\left(m_{1}+m_{2}\right)^{2}+4 m_{1} m_{2}\right]-4 m_{1} m_{2} k_{c}^{2}}{\left(m_{1} m_{2}\right)^{2}}}\right)\right]
\end{aligned}
$$


For a coupled system with an electrostatic coupling spring, the spring constant, $k_{c}$, will have a negative sign, which has been included in (5) and (6). The strength of the electrostatic coupling spring will influence the response of the amplitude ratio to a given mass perturbation, as discussed in previous works [12], with the extent of the localisation of vibration energy being greater for a system with weaker coupling.

The mass sensitivity, $S_{m}$, of a coupled resonator system is defined as the shift in the amplitude ratio at the mode frequency that is being measured as a function of the relative change in mass, $\Delta m / m$, of one of the resonators, as shown in the following expression

$$
S_{m}=\frac{\Delta\left(X_{1} / X_{2}\right)}{\Delta m / m}
$$

\section{Design and fabrication}

A dicing-free silicon-on-insulator (SOI) fabrication process [10] has been used to create a pair of closely spaced MEMS resonators that can be electrostatically coupled together. The layout of the device is shown in the schematic of Fig. 2

Starting with a $150 \mathrm{~mm}$ SOI wafer with a device layer thickness of $50 \mu \mathrm{m}$, a buried-oxide (BOX) layer thickness of $3 \mu \mathrm{m}$ and a handle layer thickness of $560 \mu \mathrm{m}$, the fabrication steps that have been performed are shown in Fig. 3 .

The resonators have been defined by deep reactive ion etching (DRIE) of $5 \mu \mathrm{m}$ trenches in the device layer, as shown in Fig. 3 $3 \mathrm{p}$, in addition to the etching of release holes that allow for the hydrofluoric (HF) acid vapour etch of the BOX that releases the resonators and the device from the wafer grid (Fig. 35). The block of the handle wafer below the resonators releases when the BOX is etched away, resulting in released resonators with no underlying substrate, eliminating the risk of stiction. Alignment of the front and back side patterns is critical in determining the duration of the HF vapour etch, with a misalignment resulting in a longer etch required to release the device. An over-etch at the anchors can affect the resonant frequency of the released structure, but with both of 


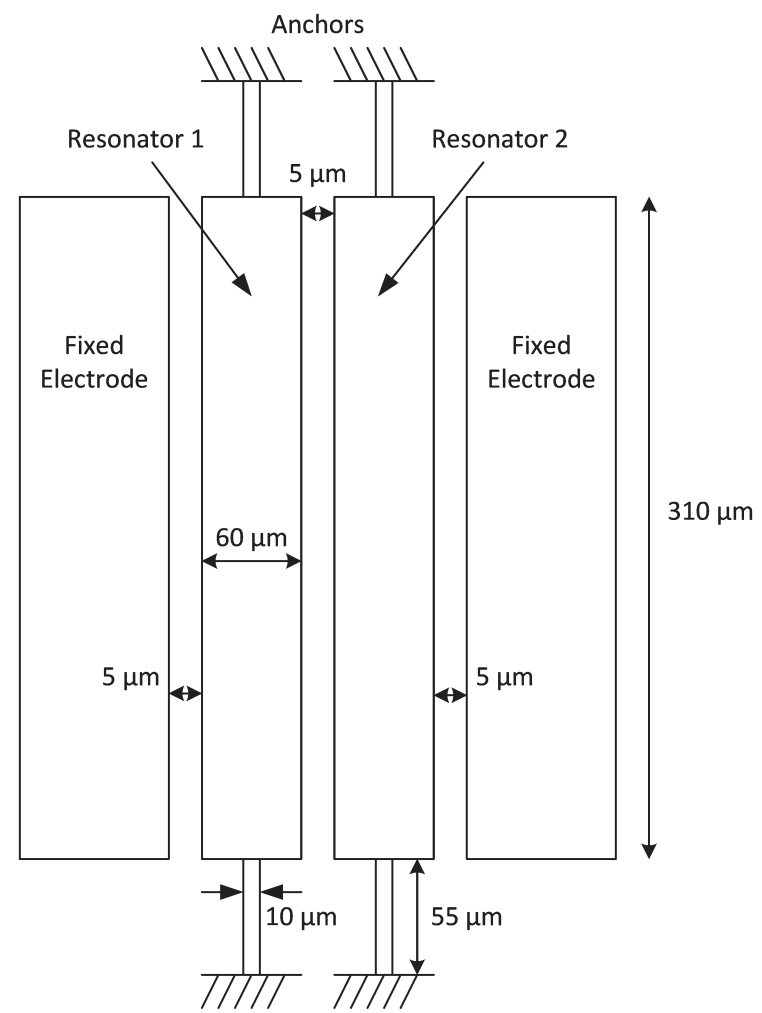

Figure 2: Schematic of coupled-resonator device. Not to scale.

the resonators in the pair being overetched equally, a misalignment of up to $10 \mu \mathrm{m}$ does not present a problem. In this work, an alignment accuracy of $2 \mu \mathrm{m}$ has been achieved. Fig. 4 shows a scanning electron micrograph (SEM) of a fabricated device.

The device consists of two resonators that are separated by a coupling gap of $5 \mu \mathrm{m}$. The resonators have anchor beams of dimensions $55 \mu \mathrm{m} \times 10 \mu \mathrm{m}$, and a wider centre section with dimensions of $60 \mu \mathrm{m} \times 310 \mu \mathrm{m}$, values that should facilitate future biological functionalisation of the surface. The thickness of the device layer is $50 \mu \mathrm{m}$. The dimensions have been chosen to ensure compliance with the design rules for the process [10, ensuring successful fabri95 cation. Parallel to each resonator, there is a fixed electrode that is separated from the movable structure by a $5 \mu \mathrm{m}$ trench. Additional DRIE of trenches has 
(a)

\begin{tabular}{c|c}
$560 \mu \mathrm{m}$ & $\begin{array}{c}50 \mu \mathrm{m} \\
\text { Device Layer } \\
\text { Handle Layer }\end{array}$ \\
$3 \mu \mathrm{m}$ BOX
\end{tabular}

(b)

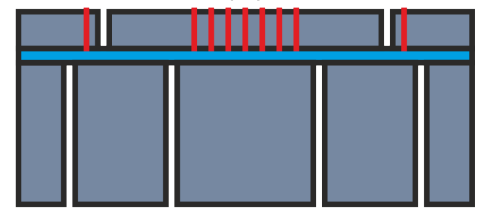

$\square$ Silicon

$\square$ BOX

$\square$ Release

(c) Holes

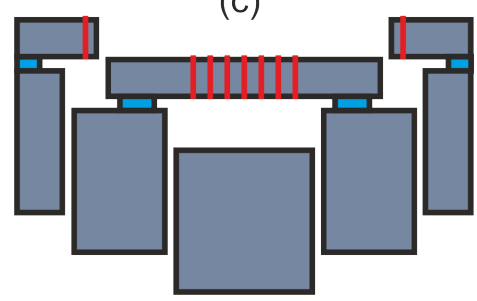

Figure 3: SOI fabrication process: (a) SOI wafer with $50 \mu \mathrm{m}$ device layer, $3 \mu \mathrm{m}$ BOX layer, and $560 \mu \mathrm{m}$ handle wafer (b) DRIE of front and back side trenches and release holes (c) HF vapour etch of BOX resulting in release of resonators and device from the wafer grid, with no stiction.

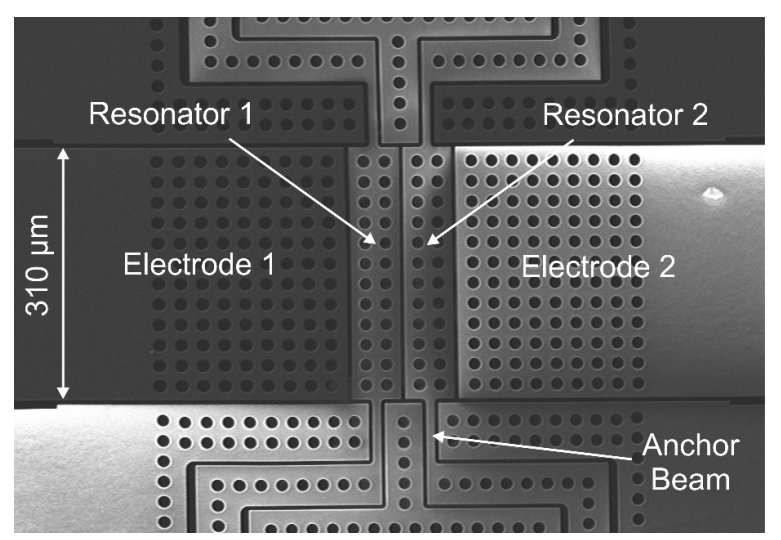

Figure 4: SEM of fabricated coupled resonator device.

been performed to create pads on the anchor areas of the device to allow for wire-bonding connections to be made to the resonators and the electrodes. 


\section{Experiment and results}

100 carrier, which has been mounted on a printed circuit board (PCB) with input and output circuitry. Fig. 5 shows a schematic of the PCB circuitry and the wirebonded connections to the coupled MEMS resonator device. An electrostatic coupling spring has been formed by DC biasing the resonators to create either beam energy of $30 \mathrm{keV}$ and milling current between $1.5 \mathrm{nA}$ and $3 \mathrm{nA}$. By visual inspection of SEM images from multiple angles, the volume of the removed silicon has been determined. The initial mass removed from resonator 1 is $2.1 \mathrm{ng}$, as shown in Fig. 6, representing a relative mass change, $\Delta m / m$, of 125

Two further sessions of milling have been performed to remove more silicon, up to a relative mass change of -0.0024 . After each mass removal, the amplitude ratio has been extracted, for $k_{c}=15.8 \mathrm{~N} / \mathrm{m}$ and $k_{c}=8.88 \mathrm{~N} / \mathrm{m}$, and the results 


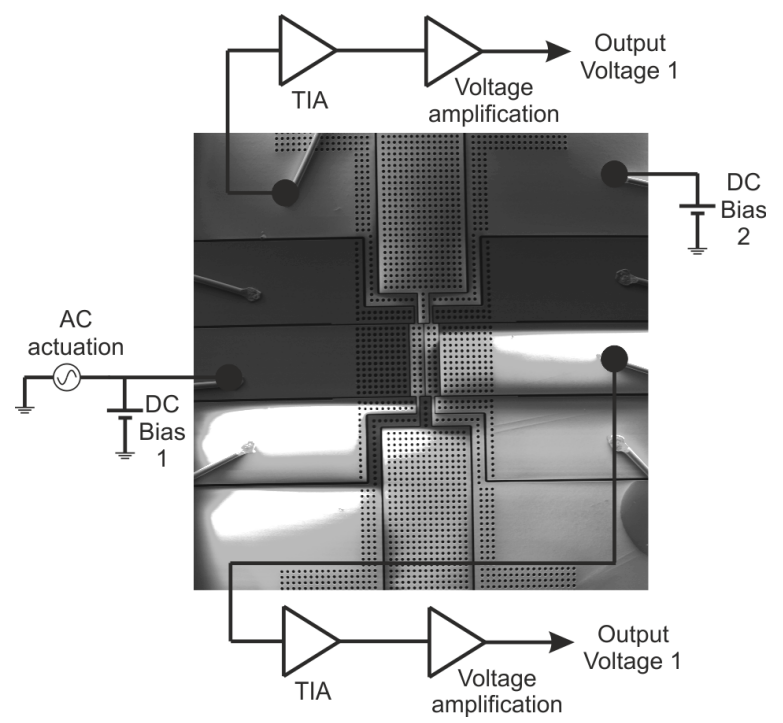

Figure 5: Experimental set-up. MEMS device has been wire-bonded to carrier positioned on printed circuit board containing output circuitry.

have been plotted in Fig. 7 .

It can be seen that the amplitude ratio response to a mass change of resonator 1 follows a linear trend, in accordance with the theoretical trend that has been calculated from (6). In addition, Fig. 7 shows that for a weaker coupling spring, the sensitivity of the amplitude ratio is increased. The sensitivity of the device has been calculated according to (7) for both values of coupling spring and is shown in Tab. 1 .

\begin{tabular}{cc}
\hline Coupling spring, $k_{c}(\mathrm{~N} / \mathrm{m})$ & Sensitivity, $S_{m}$ \\
\hline-15.8 & 12875 \\
-8.88 & 34361 \\
\hline
\end{tabular}

Table 1: Experimentally derived device sensitivities.

\section{Discussion}

The improvement in mass sensitivity that is achieved by measuring the response of the amplitude ratio of a coupled resonator pair, rather than the re- 


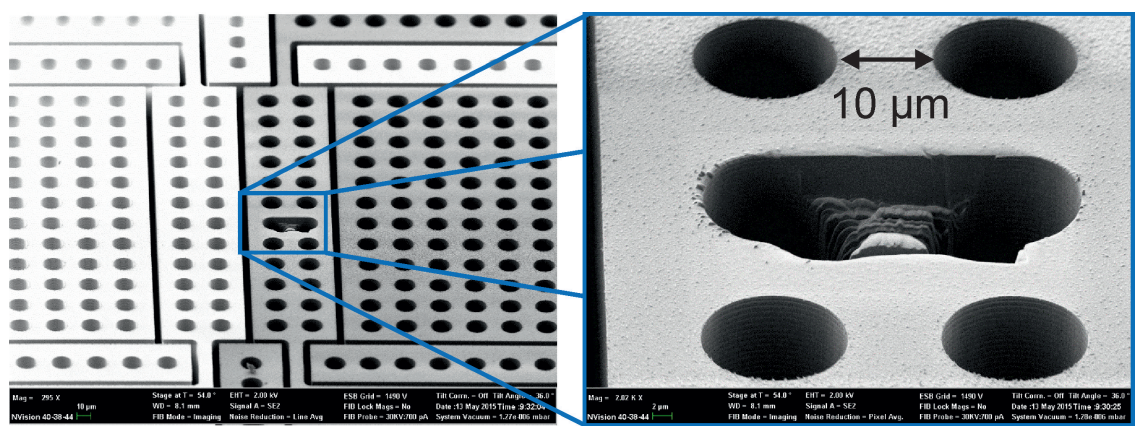

Figure 6: Initial mass removal for resonator 1 achieved using FIB milling. Visual inspection allowed for a calculation of the removed mass as $2.1 \mathrm{ng}$.

sponse of the resonant frequency of a single resonator, has been determined from the experimental results. The shift of the in-phase mode frequency of the coupled device in response to a mass change is equivalent to the resonant frequency shift of a single resonator of the same design. For a comparison to be made with the amplitude ratio response, the following definition for the frequency response sensitivity, $S_{r}$, has been used

$$
S_{r}=\frac{\Delta f_{0} / f_{0}}{\Delta m / m}
$$

Using the definition of (8) and taking the measured in-phase frequency shift of our device, the frequency response sensitivity of a single resonator of our design to a mass change has been measured to be 0.203 . The amplitude ratio mass sensitivity values of Tab. 1 show up to five orders of magnitude increase when compared to the sensitivity of the resonant frequency, validating the expected improved utility of mode-localisation based sensing with coupled resonator systems for mass sensing.

Previously reported work [9] has demonstrated mode-localised mass sensing with a sensitivity of 6367 . The sensitivity of the device characterised in this paper is up to 5.4 times greater than the state of the art, showing its potential use as a biological or chemical mass sensor. The increased sensitivity is probably a result of the higher mechanical stiffness, $k$, of the design, compared to the 


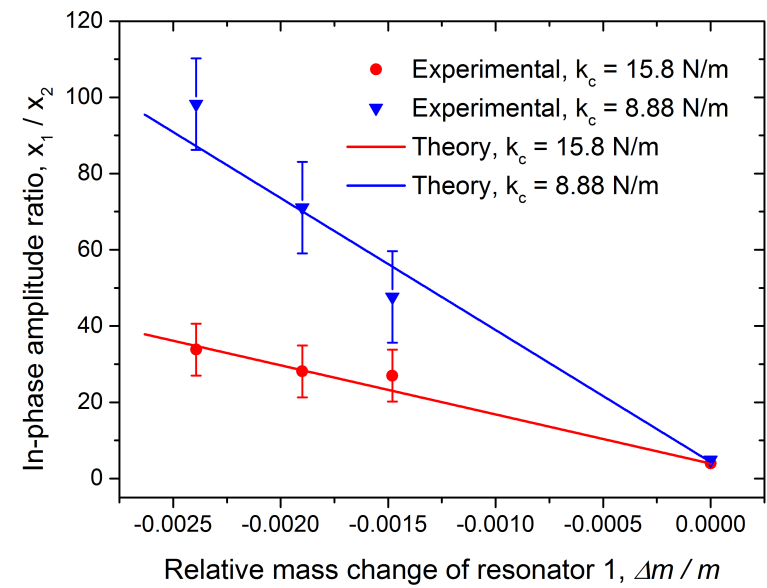

Figure 7: Theoretical and experimental response of the amplitude ratio at the in-phase mode to a mass change of resonator 1 . Responses for two different coupling spring constants are shown with error bars representing uncertainty in the mass change.

previously reported wine glass ring architecture [9], with the resulting decrease in the ratio $k_{c} / k$ leading to greater localisation of the mode shape. It has been shown that the sensitivity of the device can be tuned by simply adjusting the bias voltages applied to the resonators, altering the electrostatic coupling spring, $k_{c}$, in contrast to a device with a fixed mechanical coupling element.

\section{Conclusion}

This paper has demonstrated the mode-localisation response of a pair of electrostatically-coupled MEMS resonators to a mass perturbation. The change in the in-phase resonant mode shape has been determined by measuring the change in the vibration amplitude ratio of the two resonators in response to a mass decrease of one resonator introduced through FIB milling.

It has been shown that the sensitivity of the device is tunable through adjustment of the applied DC bias voltages, which varies the electrostatic spring strength, an advantage over a mechanically coupled device. The sensitivity of the device characterised in this work has been shown to be up to 5.4 times greater than other reported coupled MEMS resonator devices. 
Additionally, the design of the device in this work is intended to allow for its use as a biological sensor with the centre area of resonator allowing for surface

functionalisation. A further advantage of the device is the use of an established, reliable and high-yield SOI fabrication process.

\section{References}

[1] H. Zhang, E. S. Kim, Micromachined acoustic resonant mass sensor, J. Microelectromech. Syst. 14 (4) (2005) 699-706. doi:10.1109/Jmems. 2005. 845405

[2] K. L. Ekinci, X. M. H. Huang, M. L. Roukes, Ultrasensitive nanoelectromechanical mass detection, Appl. Phys. Lett. 84 (22) (2004) 4469-4471. doi:10.1063/.1.1755417.

[3] S. B. Patil, V. Chu, J. P. Conde, Mass sensing using an amorphous silicon mems resonator, Proc. Eurosens. XXIII (2009) 1063-1066doi:10.1016/j. proche.2009.07.265

[4] A. Gupta, D. Akin, R. Bashir, Single virus particle mass detection using microresonators with nanoscale thickness, Appl. Phys. Lett. 84 (11) (2004) 1976-1978. doi:10.1063/1.1667011.

[5] K. Y. Gfeller, N. Nugaeva, M. Hegner, Micromechanical oscillators as rapid biosensor for the detection of active growth of Escherichia coli., Biosens. \& Bioelectron. 21 (3) (2005) 528-533. doi:10.1016/j.bios.2004.11.018.

[6] A. P. Davila, J. Jang, A. K. Gupta, T. Walter, A. Aronson, R. Bashir, Microresonator mass sensors for detection of Bacillus anthracis Sterne spores in air and water., Biosens. \& Bioelectron. 22 (12) (2007) 3028-3035. doi:10.1016/j.bios.2007.01.012

[7] A. Gupta, J. P. Denton, H. McNally, R. Bashir, Novel fabrication method for surface micromachined thin single-crystal silicon cantilever beams, J. 
Microelectromech. Syst. 12 (2) (2003) 185-192. doi:10.1109/JMEMS. 2003. 809974

[8] P. W. Anderson, Absence of diffusion in certain random lattices, Phys. Rev. 109 (5) (1958) 1492-1505. doi:10.1103/PhysRev.109.1492.

[9] P. Thiruvenkatanathan, J. Yan, J. Woodhouse, A. Aziz, A. A. Seshia, Ultrasensitive mode-localized mass sensor with electrically tunable parametric sensitivity, Appl. Phys. Lett. 96 (8) (2010) 081913. doi:10.1063/1. 3315877 .

[10] I. Sari, I. Zeimpekis, M. Kraft, A dicing free SOI process for MEMS devices,

п. Microelectron. Eng. 95 (2012) 121-129. doi:10.1016/j.mee.2012.02. 004 .

[11] C. Zhao, G. S. Wood, J. Xie, H. Chang, S. H. Pu, M. Kraft, A force sensor based on three weakly coupled resonators with ultrahigh sensitivity, Sens.

actuators A: Phys. 232 (2015) 151-162. doi:10.1016/j.sna.2015.05. 011 .

[12] P. Thiruvenkatanathan, J. Woodhouse, J. Yan, A. A. Seshia, Manipulating vibration energy confinement in electrically coupled microelectromechanical resonator arrays, J. Microelectromech. Syst. 20 (1) (2011) 157-164. doi:10.1109/Jmems.2010.2090501 